\title{
IAEA
}

INTERNATIONAL ATOMIC ENERGY AGENCY

$22^{\text {nd }}$ IAEA Fusion Energy Conference

Geneva, Switzerland, 13 - 18 October 2008

IAEA-CN-165/OV/4-4

\section{OVERVIEW OF THE ALCATOR C-MOD RESEARCH PROGRAM*}

E.S. Marmar ${ }^{1}$, A. Bader ${ }^{1}$, M. Bakhtiari ${ }^{2}$, H. Barnard ${ }^{1}$, W. Beck ${ }^{1}$, I.

Bespamyatnov $^{3}$, A. Binus ${ }^{1}$, P. Bonoli ${ }^{1}$, B. Bose ${ }^{1}$, M. Bitter ${ }^{4}$, I. Cziegler ${ }^{1}$, G. Dekow $^{1}$, A. Dominguez ${ }^{1}$, B. Duval ${ }^{5}$, E. Edlund ${ }^{1}$, D. Ernst ${ }^{1}$, M. Ferrara ${ }^{1}$, C.

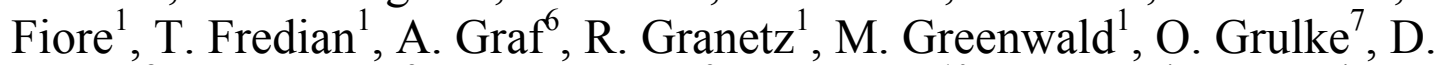
Gwinn $^{8}$, S. Harrison ${ }^{2}$, R.W. Harvey ${ }^{9}$, T. Hender ${ }^{10}$, J. Hosea ${ }^{4}$, K. Hill ${ }^{4}$, N. Howard $^{1}$, D. Howell ${ }^{10}$, A. Hubbard ${ }^{1}$, J.W. Hughes ${ }^{1}$, I. Hutchinson ${ }^{1}$, A. InceCushman $^{1}$, J. Irby ${ }^{1}$, V. Izzo ${ }^{11}$, A. Kanojia ${ }^{1}$, C. Kessel ${ }^{4}$, J.S. Ko ${ }^{1}$, P. Koert ${ }^{1}$, B.

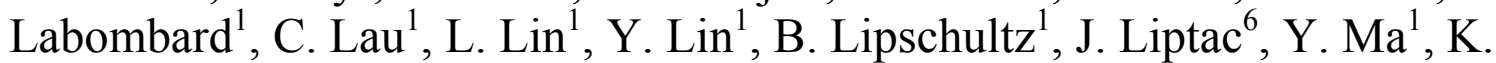
Marr $^{1}$, M. May ${ }^{6}$, R. McDermott ${ }^{1}$, O. Meneghini ${ }^{1}$, D. Mikkelsen ${ }^{4}$, R. Ochoukov', R. Parker ${ }^{1}$, C.K. Phillips ${ }^{4}$, P. Phillips ${ }^{3}$, Y. Podpaly ${ }^{1}$, M. Porkolab ${ }^{1}$, M. Reinke ${ }^{1}$, J. Rice ${ }^{1}$, W. Rowan ${ }^{3}$, S. Scott ${ }^{4}$, A. Schmidt ${ }^{1}$, J. Sears ${ }^{1}$, S. Shiraiwa ${ }^{1}$, A.C.C. Sips $^{7}$, N. Smick ${ }^{1}$, J. Snipes ${ }^{12}$, J. Stillerman ${ }^{1}$, Y. Takase ${ }^{13}$, D. Terry ${ }^{1}$, J. Terry ${ }^{1}$, N.

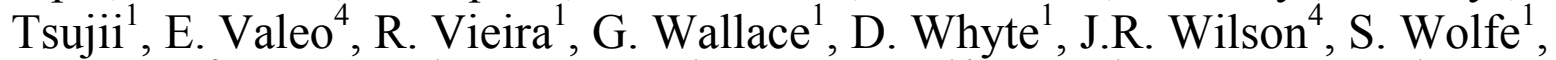

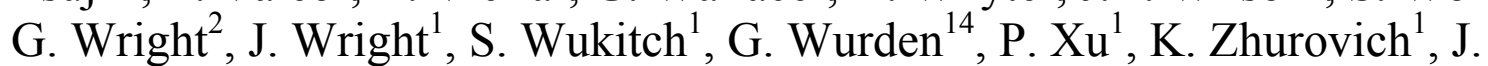
Zaks $^{1}$, S. Zweben ${ }^{4}$

MIT Plasma Science and Fusion Center, 77 Mass. Avenue, Cambridge, MA 02139 USA *Research supported by the U.S. Department of Energy.

\footnotetext{
${ }^{1}$ Massachusetts Institute of Technology, Cambridge, MA USA

${ }^{2}$ University of Wisconsin, Madison, WI USA

${ }^{3}$ Univerisity of Texas, Austin, TX USA

${ }^{4}$ Princeton Plasma Physics Laboratory, Princeton, NJ USA

${ }^{5}$ EPFL, Lausanne, Switzerland

${ }^{6}$ Lawrence Livermore National Laboratory, Livermore, CA USA

${ }^{7}$ Institute for Plasma Physics, Garching, Germany

${ }^{8}$ Bagley Associates, Lowell, MA USA

${ }^{9}$ CompX Corporation, Del Mar, CA USA

${ }^{10}$ EURATOM/UKAEA Fusion Association, Culham Science Centre, Abingdon, UK

${ }^{11}$ University of California, San Diego, CA USA

${ }^{12}$ ITER International Organization, Cadarache, France

${ }^{13}$ University of Tokyo, Tokyo, Japan

${ }^{14}$ Los Alamos National Laboratory, Los Alamos, NM USA
}

This is a preprint of a paper intended for presentation at a scientific meeting. Because of the provisional nature of its content and since changes of substance or detail may have to be made before publication, the preprint is made available on the understanding that it will not be cited in the literature or in any way be reproduced in its present form. The views expressed and the statements made remain the responsibility of the named author(s); the views do not necessarily reflect those of the government of the designating Member State(s) or of the designating organization(s). In particular, neither the IAEA nor any other organization or body sponsoring this meeting can be held responsible for any material reproduced in this preprint. 


\title{
Overview of the Alcator C-Mod Research Program
}

E.S. Marmar, A. Bader, M. Bakhtiari, H. Barnard, W. Beck, I. Bespamyatnov, A. Binus, P. Bonoli, B. Bose, M. Bitter, I. Cziegler, G. Dekow, A. Dominguez, B. Duval, E. Edlund, D. Ernst, M. Ferrara, C. Fiore, T. Fredian, A. Graf, R. Granetz, M. Greenwald, O. Grulke, D. Gwinn, S. Harrison, R.W. Harvey, T. Hender, J. Hosea, K. Hill, N. Howard, D. Howell, A. Hubbard, J.W. Hughes, I. Hutchinson, A. Ince-Cushman, J. Irby, V. Izzo, A. Kanojia, C. Kessel, J.S. Ko, P. Koert, B. Labombard, C. Lau, L. Lin, Y. Lin, B. Lipschultz, J. Liptac, Y. Ma, K. Marr, M. May, R. Mcdermott, O. Meneghini, D. Mikkelsen, R. Ochoukov, R. Parker, C.K. Phillips, P. Phillips, Y. Podpaly, M. Porkolab, M. Reinke, J. Rice, W. Rowan, S. Scott, A. Schmidt, J. Sears, S. Shiraiwa, A.C.C. Sips, N. Smick, J. Snipes, J. Stillerman, Y. Takase, D. Terry, J. Terry, N. Tsujii, E. Valeo, R. Vieira, G. Wallace, D. Whyte, J.R. Wilson, S. Wolfe, G. Wright, J. Wright, S. Wukitch, G. Wurden, P. Xu, K. Zhurovich, J. Zaks, S. Zweben

e-mail contact of the main author: marmar@psfc.mit.edu

\begin{abstract}
This paper summarizes highlights of research results from the Alcator C-Mod tokamak covering the period 2006 through 2008. Active flow drive, using mode converted waves in the ion cyclotron range of frequencies (ICRF), has been observed for the first time in a tokamak plasma, using a mix of D and $3 \mathrm{He}$ ion species; toroidal and poloidal flows are driven near the location of the mode conversion layer. ICRF induced edge sheaths are implicated in both the erosion of thin boron coatings and the generation of metallic impurities. Lower Hybrid RF has been used for efficient current drive, current profile modification, and toroidal flow drive. In addition, LHRF has been used to modify the H-mode pedestal, increasing temperature, decreasing density, and lowering the pedestal collisionality. Studies of hydrogen isotope retention in solid metallic plasma facing components reveal significantly higher retention than expected from ex-situ laboratory studies; a model to explain the results, based on plasma/neutral induced lattice damage has been developed and tested. During gaspuff mitigation of disruptions, induced MHD causes the magnetic field to become stochastic, resulting in reduction of halo currents, spreading of plasma power loading, and loss of run-away electrons before they cause damage. Detailed pedestal rotation profile measurements have been used to infer ER profiles, and correlation with global H-mode confinement. An improved L-mode regime, obtained at $\mathrm{q}_{95} \leq 3$ with ion drift away from the active x-point, shows very good confinement without a strong density pedestal, and no evidence of particle or impurity accumulation without the need for ELMs or any other edge density regulation mechanism.
\end{abstract}

\section{Introduction}

Research on the Alcator C-Mod tokamak focuses on the plasma physics and plasma engineering required for ITER and for attractive fusion reactors. C-Mod is prototypical of ITER in several key respects including high toroidal magnetic field, equilibrated ions and electrons, high plasma density, high power density in the scrape off layer (SOL), low momentum input, high-Z plasma facing components (PFCs), divertor neutral and photon opacity, long pulse length compared to the skin time, and the ability to run identically shaped, diverted configurations. Recent upgrades to the facility include the addition of a divertor cryopump for density control, a $3 \mathrm{MW}$ source Lower Hybrid Current Drive (LHCD) microwave system, and new and improved diagnostic systems, particularly for profile measurements and fluctuations. Recent experimental campaigns have focused on current and flow drive, RF impurity generation, deuterium retention, mitigation of runaway electron production during disruptions, H-mode dynamics and pedestal control, and scrape-off-layer flows and transport. We also report on an improved L-mode regime, which combines good energy confinement with an absence of impurity accumulation.

\section{Mode Conversion Flow Drive}




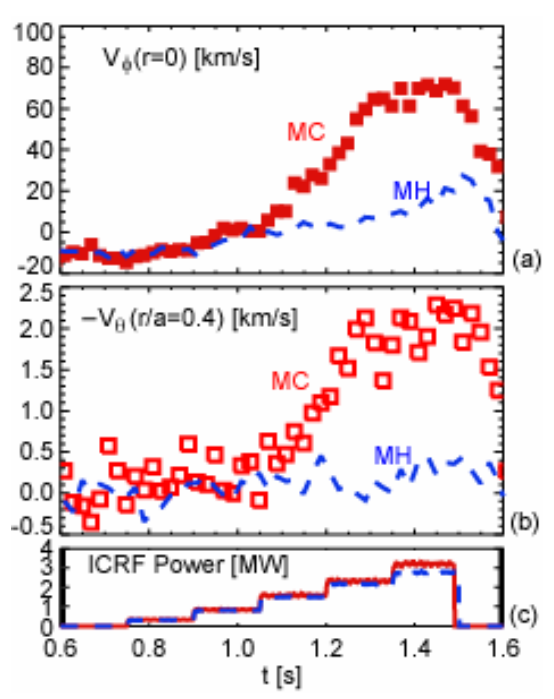

Figure 1. (a) Central toroidal rotation; (b) Polodial rotation at $r / a=0.4$; (c) RF power traces. $(M C$ - mode conversion, and $M H$ - minority heating).
Flow drive via externally launched electromagnetic waves has been widely identified as a high leverage tool that, if successful, can produce great benefits. For the first time in tokamak plasmas, we have demonstrated plasma flow drive, in both toroidal $\left(\mathrm{V}_{\phi}\right)$ and poloidal $\left(\mathrm{V}_{\theta}\right)$ directions, by an ion cyclotron range of frequencies (ICRF) mode conversion process. $\left.{ }^{[1}\right]$ Our result suggests the potential of driving significant rotation and flow shear on ITER using ICRF power, and offers a unique means for manipulating transport via flow shear stabilization in reactor relevant regimes.

In figure 1, we compare the time traces of $\mathrm{V}_{\phi}$ and $\mathrm{V}_{\theta}$ of two plasmas: one with ICRF minority heating $(\mathrm{MH})$ and the other with ICRF mode conversion (MC). The velocities are measured by high resolution $\mathrm{x}$-ray spectroscopy of $\mathrm{Ar}^{16+}$ and $\mathrm{Ar}^{17+}$ impurities. Both plasmas are upper-single-null L-mode plasmas with $\mathrm{I}_{\mathrm{p}}=$ $0.8 \mathrm{MA}, \mathrm{B}_{\mathrm{t} 0}=5.1 \mathrm{~T}$ and $<\mathrm{n}_{\mathrm{e}}>\approx 1.2 \times 10^{20} \mathrm{~m}^{-3}$. The $\mathrm{MH}$ plasma is heated by ICRF power at $80 \mathrm{MHz}$ with a fundamental $\mathrm{H}$ ion cyclotron resonance near axis. The

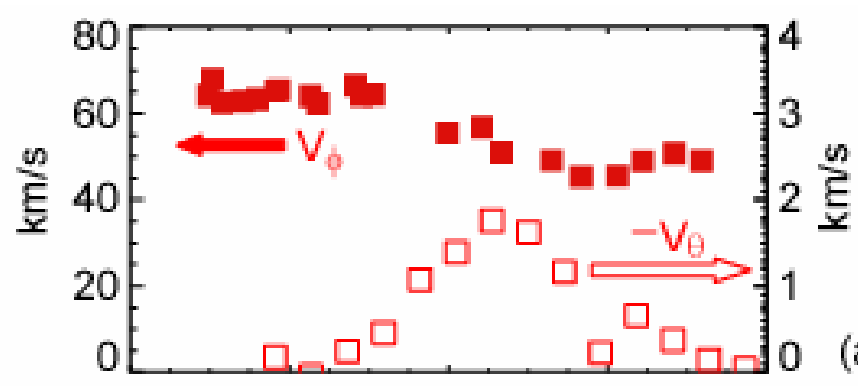

(a)
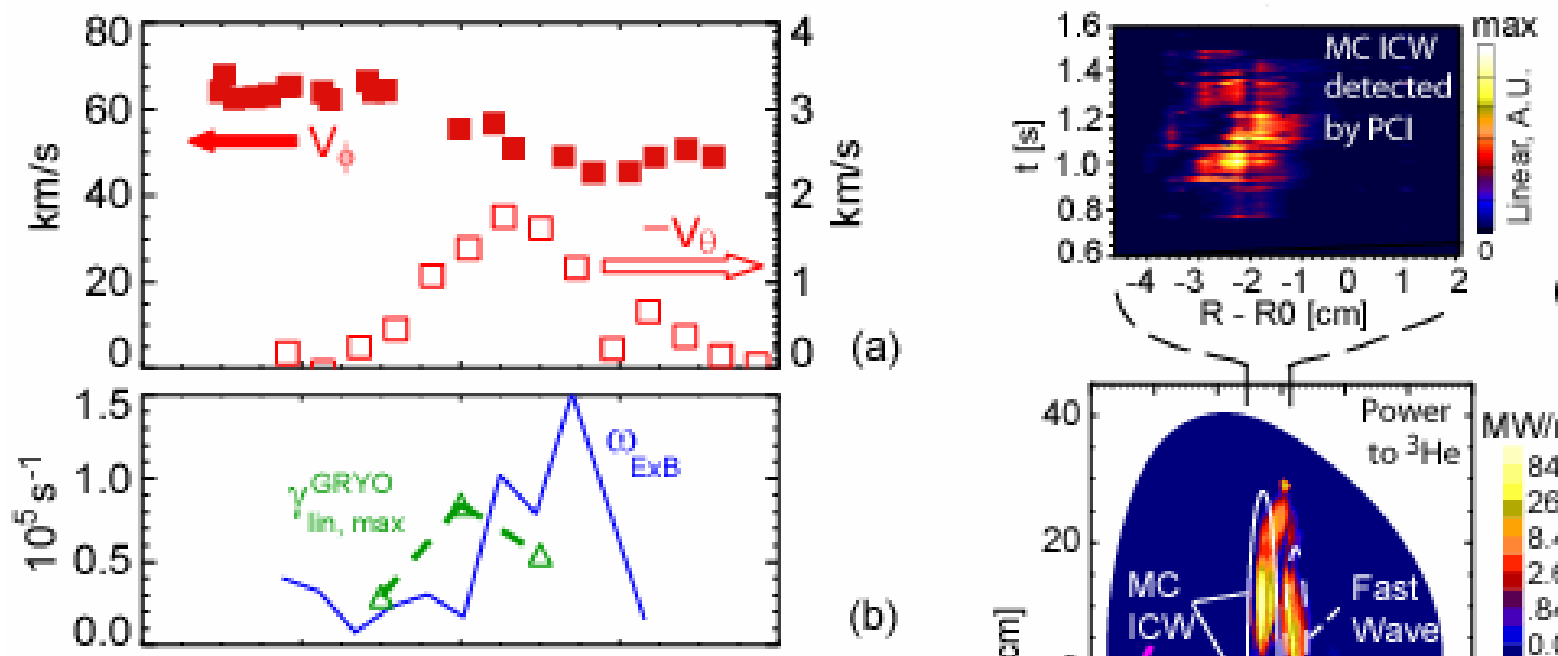

(d)

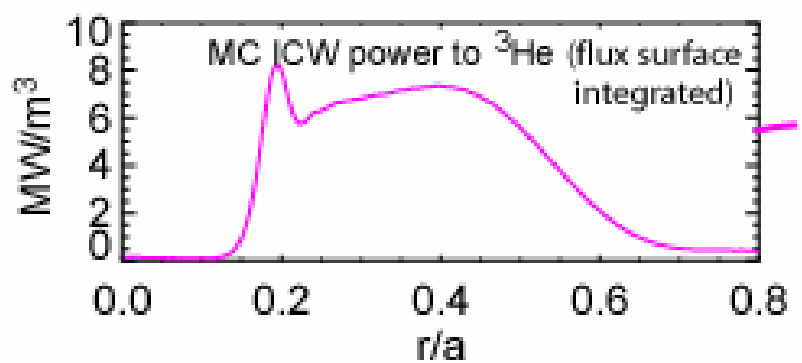

(b)

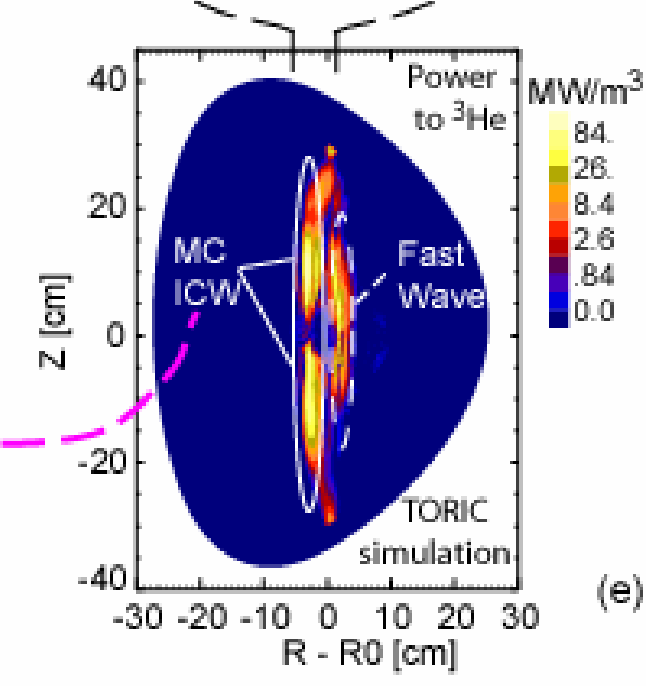

Figure 2: (a) Rotation profiles at $t=1.4$ sec of the MC plasma in Fig. ; (b) $\omega_{E \times B}$ compared to $\gamma_{\text {lin, max }}$ from GYRO simulation; (c) Power deposition to ${ }^{3} \mathrm{He}$ ions from TORIC; (d) MC ICW in R and tetected by heterodyne PCI; (e) Power deposition in 2-D by TORIC simulation.

MC plasma is a mixture of $\mathrm{D}$ and ${ }^{3} \mathrm{He}$, with $\mathrm{n}_{\mathrm{He}-3} / \mathrm{n}_{\mathrm{e}} \sim 0.1$, and the rf frequency is at $50 \mathrm{MHz}$. The launched fast wave undergoes mode conversion at the $\mathrm{D}-{ }^{3} \mathrm{He}$ hybrid layer near the magnetic axis. Figure 2-(a) and (b) show that in the $\mathrm{MC}$ plasma $\mathrm{V}_{\phi}(\mathrm{r}=0)$ (co-current direction) and $\mathrm{V}_{\theta}(\mathrm{r} / \mathrm{a}=0.4)$ (ion diamagnetic direction) rise to substantially higher levels than 
those in the MH plasma at the same rf power traces (figure 2-(c)). $\mathrm{V}_{\phi}$ in these MC plasmas is found to increase with the $\mathrm{rf}$ power $(\sim 30 \mathrm{~km} / \mathrm{s}$ per MW input power). Toroidal phasing of the antenna also plays some role in that the velocity is largest in co-current phase, followed by dipole and counter-current phases. In all phases the velocity changes exceed the empirical scaling for the intrinsic toroidal rotation $\left(\propto \Delta \mathrm{W} / \mathrm{I}_{\mathrm{p}}\right)$ by at least a factor of 2 .

The RF flow drive results in a broad, centrally peaked $\mathrm{V}_{\phi}$ profile, and a locally peaked $\mathrm{V}_{\theta}$ profile as shown in Figure 2-(a). The radial $E_{r}$ has been calculated from the measured flow velocities and the radial force balance equation, and a strong $\mathrm{E} \times \mathrm{B}$ shear, defined as $\omega_{E \times B} \approx\left(R^{2} B_{\theta}^{2} / B\right)(\partial / \partial \psi)\left(E_{r} / R B_{\theta}\right)$, is found to exist in the region of $0.45<\mathrm{r} / \mathrm{a}<0.55$. As shown in figure 2-(b), the $\mathrm{E} \times \mathrm{B}$ shearing rate is comparable to $\gamma_{\operatorname{lin}, \max }^{\mathrm{GYR}}$, the maximum ITG growth rate obtained from linear GYRO simulation.

Mode converted ion cyclotron waves (MC ICW) are detected in the MC plasma by heterodyne Phase Contrast Imaging (PCI)[2] near the magnetic axis on the high field side (shown in figure 2-(d)). Figure 2-(e) shows the 2-D partition of the rf power deposition to ${ }^{3} \mathrm{He}$ ions by the MC ICW and by the fast wave from full wave TORIC simulation. Having a much larger $\mathrm{k}_{\|}$than the fast wave $\left(\sim 50 \mathrm{~m}^{-1}\right.$ vs. $\left.\sim 10 \mathrm{~m}^{-1}\right)$, the MC ICW can be strongly damped by the ${ }^{3} \mathrm{He}$ ions via the Doppler broadened IC resonance, $\omega=\omega_{c,{ }^{3} \mathrm{He}}+k_{\|} v_{t i}$, near the MC layer. Figure 2-(c) shows the flux surface integrated power deposition of the MC ICW to ${ }^{3} \mathrm{He}$ ions, indicating a broad peak in the region of observed poloidal flow. Being a slow and

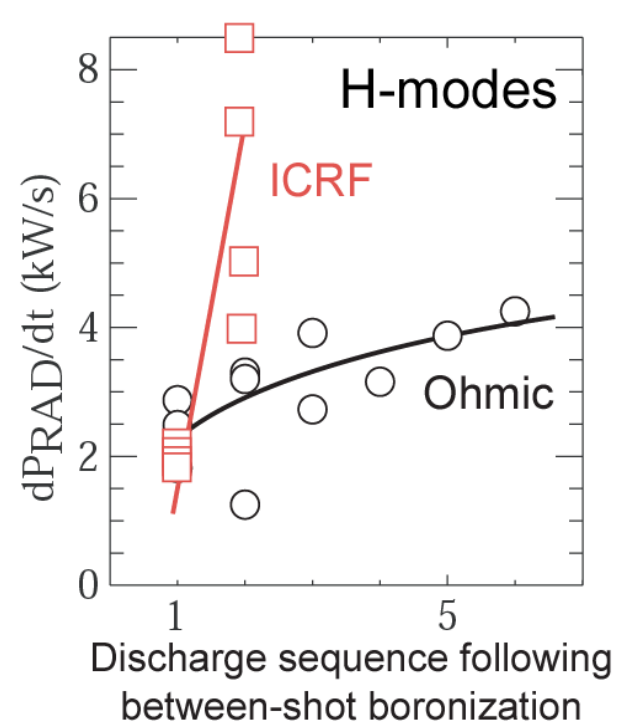

Figure 3. Rates of radiated power increase following the L to H mode transition, as a function of shot number following between-shot boronizations. The data compare ICRF heated discharges with ohmic heating alone. In all cases, the total input energy to the discharges is the same. short wavelength wave, similar to direct launched ion Bernstein wave (IBW), the MC ICW has been suggested for rf flow drive in a previous numerical study $\left[{ }^{3}\right]$. The flow velocities in our experiment are comparable to the estimates from Ref. $\left[{ }^{4}\right]$ if we assume the same wave-particle interaction mechanism as IBW.

ITER has a similar B field and ICRF frequency to those in our MC experiment on Alcator C-Mod. A recent simulation study has shown the existence of the MC ICW on ITER D-T- ${ }^{3} \mathrm{He}$ plasmas $\left[{ }^{5}\right]$. As a result, we expect the same MC flow drive mechanism to be applicable on ITER, and potentially drive strong rotation and flow shear.

\section{ICRF Impurity Sources and Sheaths}

Detailed studies on Alcator C-Mod have been aimed at characterizing and understanding the generation of high $\mathrm{Z}$ impurities during high power ICRF heating experiments, looking particularly at the role that RF sheaths play.[ ${ }^{6}$ ] C-Mod has characteristics that are similar in many respects to conditions expected in ITER. The C-Mod ICRF antennas obtain power densities $\left(10 \mathrm{MW} / \mathrm{m}^{2}\right)$ in excess of those planned for ITER. The 
RF wave single-pass absorption is similar; thus, the RF fields in both C-Mod and ITER will be localized toroidally. Furthermore, CMod utilizes solid high-Z (molybdenum) PFCs. The scrape off layer is also opaque to neutrals in C-Mod, as expected on ITER, an important consideration for impurity transport. In addition to the high $\mathrm{Z}$ PFCs being considered for ITER, beryllium has been proposed to cover most of the first wall and the ICRF faraday screen. In C-Mod, we routinely coat the PFCs with a low-Z boron film in-situ $\left[{ }^{7}\right]$, using 'boronization' allowing an opportunity to investigate the compatibility of high power ICRF and low-Z films.

Direct comparisons of impurity influx and radiated power between ICRF heated and ohmic H-mode plasmas, with comparable total input energy, show that ICRF is dominant. Figure 3, shows such a comparison, with increase in radiated power plotted as a function of plasma shot number following a between-shot boronization, which deposits a thin layer $(\sim 20 \mathrm{~nm})$ of boron on the Mo PFCs. Emissive probe measurements prove that the RF sheaths are tied to the active ICRF antenna along magnetic field lines (figure 4). These probes consist of thin thoriated tungsten wire maintained at $\sim 1800{ }^{\circ} \mathrm{C}$ where the emitted electron current is greater than the free streaming electron flux. The floating potential of the

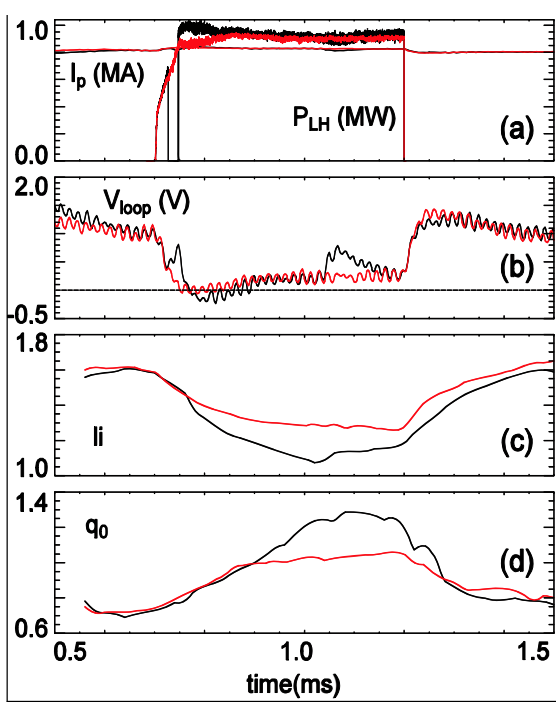

Figure 5. Time evolution of two LH discharges showing reduction in loop voltage, decrease in plasma inductance and increase in central safety factor. $n_{\|}=1.5$ (black), $n_{\|}=$ 1.9 (red)

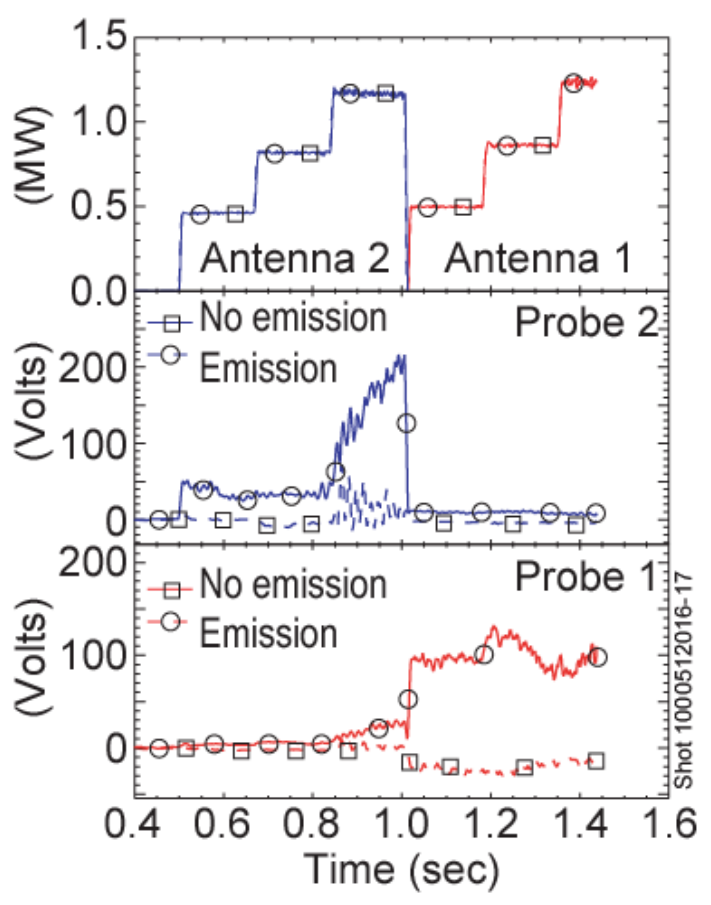

Figure 4. ICRF sheath enhancement measured using emissive probes. The increase in potential appears only on the probe which is magnetically connected to the active ICRF antenna. heated filament is a measure of the plasma potential to within approximately \pm Te. $\left[{ }^{8}, 9\right]$ Converting the antenna protection tiles from Mo to boron-nitride had no effect on Mo contamination, proving that these nearby structures are not the dominant impurity sources.

By varying the deposition location of the boron during the boronization process, we have determined that the most important impurity source locations for plasma contamination are outboard of the divertor in major radius, with poloidal limiters and the shelf beyond the divertor being the prime suspects. For the upcoming (2009) experimental campaign, all of these Mo surfaces will have a thick $(\sim 100 \mu \mathrm{m})$ boron coating applied, to investigate if that can reduce or eliminate Mo influx during application of high power ICRF. 


\section{Lower Hybrid Current and Flow Drive}

Non-inductive sustainment of plasma current is required for steady state operation of the tokamak. In ITER, the second phase of D-T operations will be aimed at quasi-steady advanced scenarios, using a combination of bootstrap, RF and beam current drive. Because Lower Hybrid is the most efficient of the auxiliary current drive approaches, its inclusion on ITER for this second phase may be critical. C-Mod, operating at the same magnetic field and density as ITER, as well as with a lower hybrid system at about the same frequency as that envisioned for ITER, is in a unique position to provide critical data to inform the Lower Hybrid decision on ITER. Significant progress was made over the last two years in the area of LHCD on C-Mod. At intermediate plasma densities $\left(\sim 5 \times 10^{19} \mathrm{~m}^{-3}\right)$, close to $100 \%$ of the plasma current has been sustained using up to $1.2 \mathrm{MW}$ of net power to the plasma $(\mathrm{f}=4.6 \mathrm{GHz})$, coupled through an 88 window phased array launcher. Plasma parameter time histories for two discharges, where the loop voltage was held close to 0 for about 2 resistive skin times, are shown in figure 5. The results with two different $\mathrm{n}_{\|}$spectra are compared.

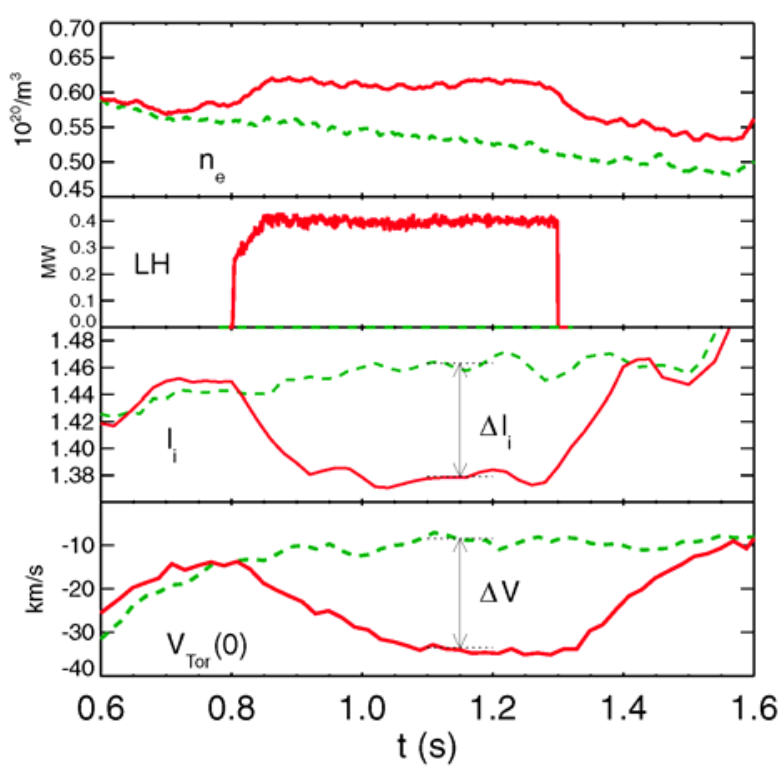

Figure 6. Electron density, LHCD power, internal inductance and core rotation velocity for discharges with (red) and without (green dashed) LHCD.

MSE measurements of current density profile, corroborated by imaging of hard x-rays due to the LH driven epithermal electrons, epithermal electrons, in agreement with expectations based on the ray tracing code GENRAY coupled with the Fokker-Planck code CQL3D $\left[{ }^{10}\right]$. Simulations using a full wave code are underway.

Another issue for future tokamak reactors is the generation and control of rotation velocity profiles for suppression of destructive magnetohyrodynamic oscillations, in the absence of external momentum input. Following application of LHRF power, the core rotation in Alcator C-Mod plasmas is found to increment in the direction opposite to the plasma current, $\left[{ }^{11}\right.$ in conjunction with a decrease in the plasma

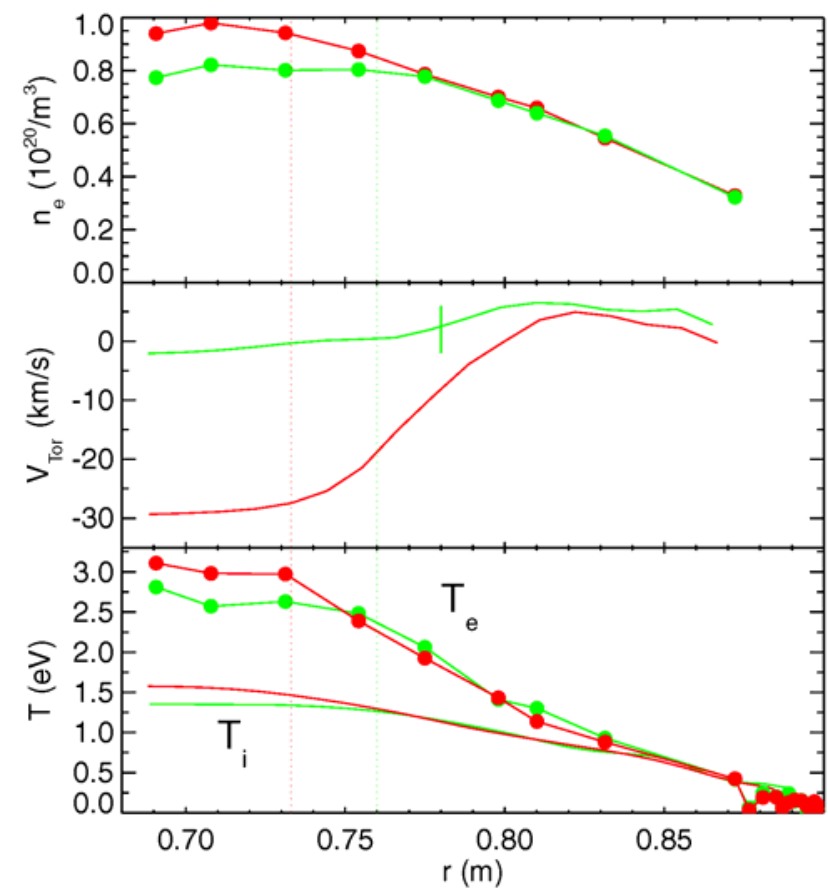

Figure 7. The electron density, rotation velocity and temperature profiles before (green) and during (red) LHCD. 
internal inductance, which is indicative of a broadening of the current density profile. Shown in figure 6 is a comparison of two discharges, with and without LHRF power. Along with the

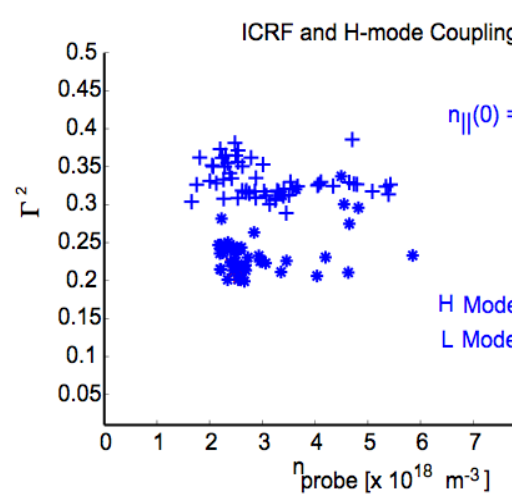

Figure 8. Comparisons of reflected power between $L$ - and $H$ - mode plasmas as a function of plasma density at the $\mathrm{LH}$ launcher mouth. decreases in the internal inductance and the core rotation velocity, there is peaking of the electron and impurity density profiles, as well as of the ion and electron temperature profiles, as shown in figure 7 . The mechanism generating the counter-current rotation is currently unknown, but it is consistent with an inward shift of energetic electron orbits, giving rise to a negative core radial electric field. The peaking in the density, rotation (in the counter-current direction) and temperature profiles occurs over a time scale similar to the current relaxation time but slow compared to the energy and momentum confinement times. The magnitudes of the changes in the internal inductance and the central rotation velocity are correlated and found to increase with increasing microwave power and decreasing electron density.

Future experiments will attempt to exploit this current drive in the higher density plasmas relevant for fusion reactors, to manipulate the peaking of the density and temperature profiles in a regulated fashion, and to exploit this new tool for control of the flow velocity profile.

\section{Pedestal Modification with Lower Hybrid Waves}

Experiments have begun on C-Mod to investigate the coupling and propagation of lower hybrid RF through the H-mode edge pedestal, a prerequisite for development and exploitation of LHCD for sustainment of advanced scenarios with an edge confinement barrier. Since the H-mode edge has significantly steeper density gradients (scale length of only a few $\mathrm{mm}$ on C-Mod), propagation of the waves can be a concern. $\left[{ }^{12}\right]$ Initial C-Mod results are very encouraging in this regard, with lower reflection coefficients than in typical L-mode conditions (see figure 8).

A new observation from these experiments is that the LHRF can have a strong effect on the pedestal itself, increasing the temperature pedestal and decreasing the pedestal density, while keeping the pedestal pressure constant. Figure 9 shows comparisons of the pedestal profiles with and without LHCD; the resulting pedestal collisionality is substantially reduced. We have yet to determine the mechanism for this effect and the range of operational conditions over which it can be exploited, but it suggests a new major opportunity for external control of the H-mode pedestal. Further research will investigate the underlying physics of particle and energy transport in the pedestal, and explore the potential for H-mode

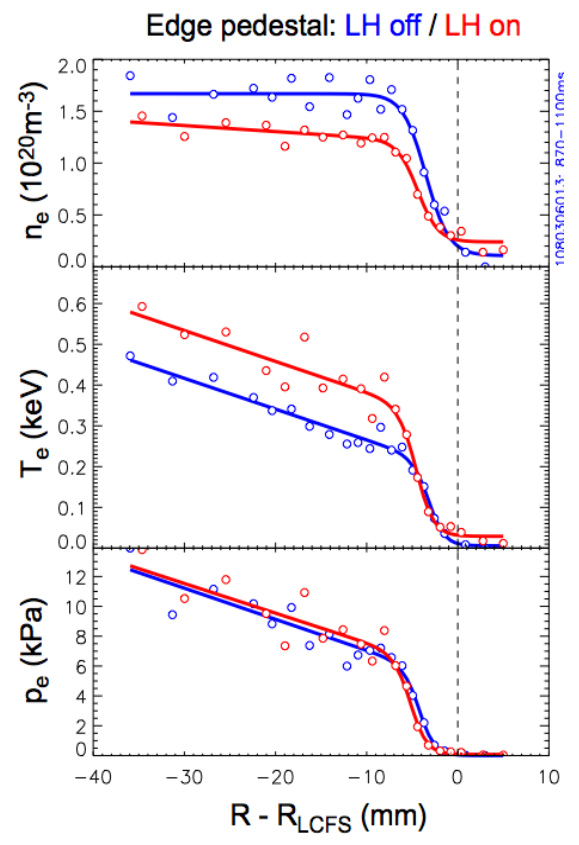

Figure 9. Lower Hybrid RF can be used to modify the H-mode pedestal, decreasing density and increasing temperature. The pedestal pressure is increased slightly, while the collisionality is substantially reduced. 
confinement optimization and ELM control/mitigation. LH power upgrades planned for 2009-2010 should enable substantial current profile modification in H-mode plasmas.

\section{Hydrogen Isotope Retention}

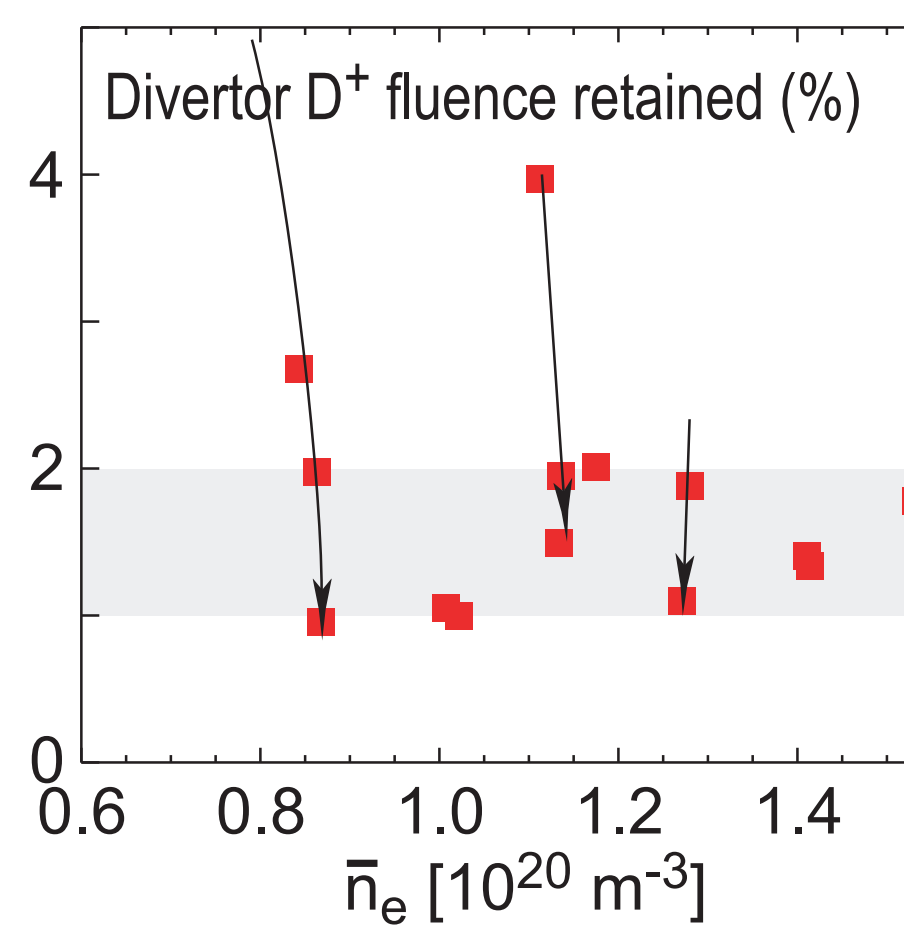

Tritium has to be tightly controlled in ITER and in reactors for reasons of safety and economics. High-Z refractory metals like tungsten and molybdenum are favored over carbon for reactor PFCs due to their resistance to erosion and nuclear degradation. Only a small fraction of the fuel flowing through the reactor will be burned in each pass; most will impact on heat-bearing surfaces (plasma facing components or 'PFCs'). If more than $10^{-7}$ of the impacting fuel ions become stuck in the PFCs, then tritium will be stored faster than it can be bred. For ITER, a pulsed experiment, the requirement for low retention in the walls is eased slightly, to about $10^{-6}$. Alcator C-Mod operates with all PFC components fabricated of solid molybdenum and tungsten, unique among divertor tokamaks. Over several run campaigns a new accurate method of global retention of deuterium during plasma discharges has been developed. $\left[{ }^{13}\right]$ The global retention in Alcator C-Mod has been related back to the measured ion fluences to surfaces. The study shows that of order $1 \%$ of the incident deuterium ions are retained in surfaces during a single C-Mod discharge (figure 10) independent of plasma discharge density and thus incident ion flux density $\left(\# / \mathrm{m}^{2} / \mathrm{s}\right)$. Additionally, the retention does not appear to saturate over consecutive discharges, the total fluence of which approached that expected from a single ITER discharge. Since an undisturbed molybdenum lattice can only accept a low-level of D residing at inherent distortions in the lattice, so-called 'traps', the retention should be limited by diffusion in the material lattice to those traps and the retention should scale with the square root of fluence. The C-Mod result of retention scaling linearly with fluence points towards either expansion of existing traps or creation of new traps.

It has been postulated that the high fluxes of ions implanted very short distances into the material $(1 \mathrm{~nm})$ lead to local neutral densities approaching that of the lattice. As the tritium collects, the pressure becomes high enough to distort the lattice, creating and enlarging traps and allowing more tritium to be stored. Deuterium (and the other hydrogen isotopes) can only be released from a surface after recombining into molecules, a relatively slow process. For helium, an inert gas, release from a surface is not limited by recombination which suggested a test for the model. C-Mod experiments showed that retention is strongly reduced by the use of helium compared to deuterium, supporting the model that surface 
recombination is a rate-limiting process. While single discharge retention is high, we also find that major disruptions result in significant release of trapped deuterium. Based on surface analysis of tiles removed from the vessel following a 15 week experimental campaign, the long-term retention (integrated over a campaign) is $1000 \mathrm{x}$ less than that found for a single non-disruptive discharge.

These results indicate that in order for ITER and reactors utilizing tungsten PFCs to keep the $\mathrm{T}$ retention low they will either have to operate with PFCs at high temperature, $800-1000^{\circ} \mathrm{C}$ (not an option for ITER) to enhance diffusion of $\mathrm{T}$ within the lattice and recombination at the surface, or build in some intermittent method of heating the plasma facing components.

\section{Disruption Mitigation}

Major disruptions are a concern for ITER for at least three reasons: forces on structures due to eddy and halo currents; extremely high surface power loading which can lead to melting; and generation of runaway electrons. While relatively few in number, runaways can cause severe localized damage when they eventually impact material surfaces. It has been established on C-Mod $\left[{ }^{14}\right]$ and elsewhere $\left[{ }^{15}\right]$ that intentional rapid cooling by gas jet impurity injection, which forces the plasma to safely radiate away its energy, is highly successful at mitigating halo currents and surface power loading, and is needed for ITER. However, runaway generation has been more difficult to evaluate.

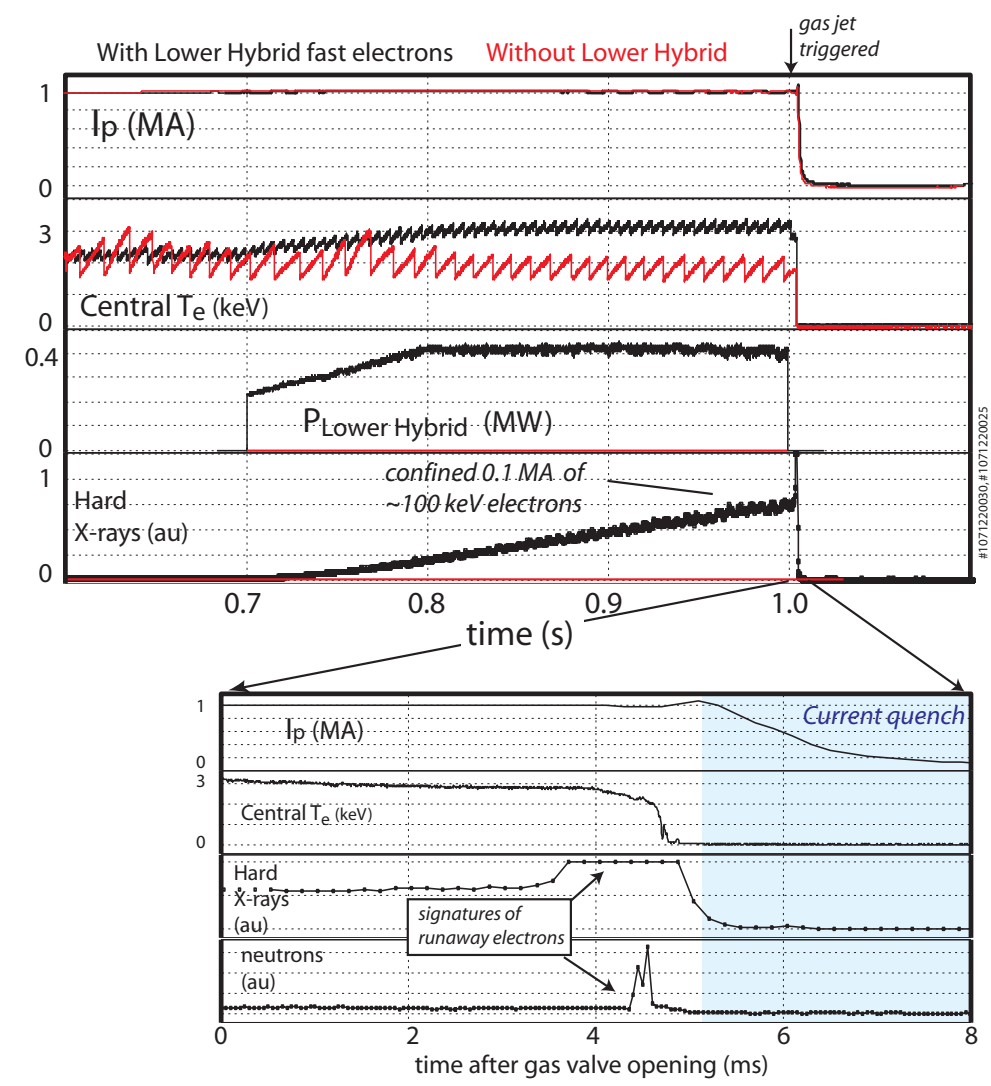

Figure 11. C-Mod gas jet injection rapidly terminates plasma by safe radiation and existing $\mathrm{LH}$-driven suprathermal electrons are promptly lost so that they cannot be amplified when the current decays and electric field is large. 
Recent experiments and modeling on Alcator C-Mod indicate a possible solution to the problem: during the thermal quench, strong MHD modes grow large enough to cause the magnetic fields to become stochastic, in turn causing prompt loss of fast electrons, and suppressing runaway amplification. A key challenge
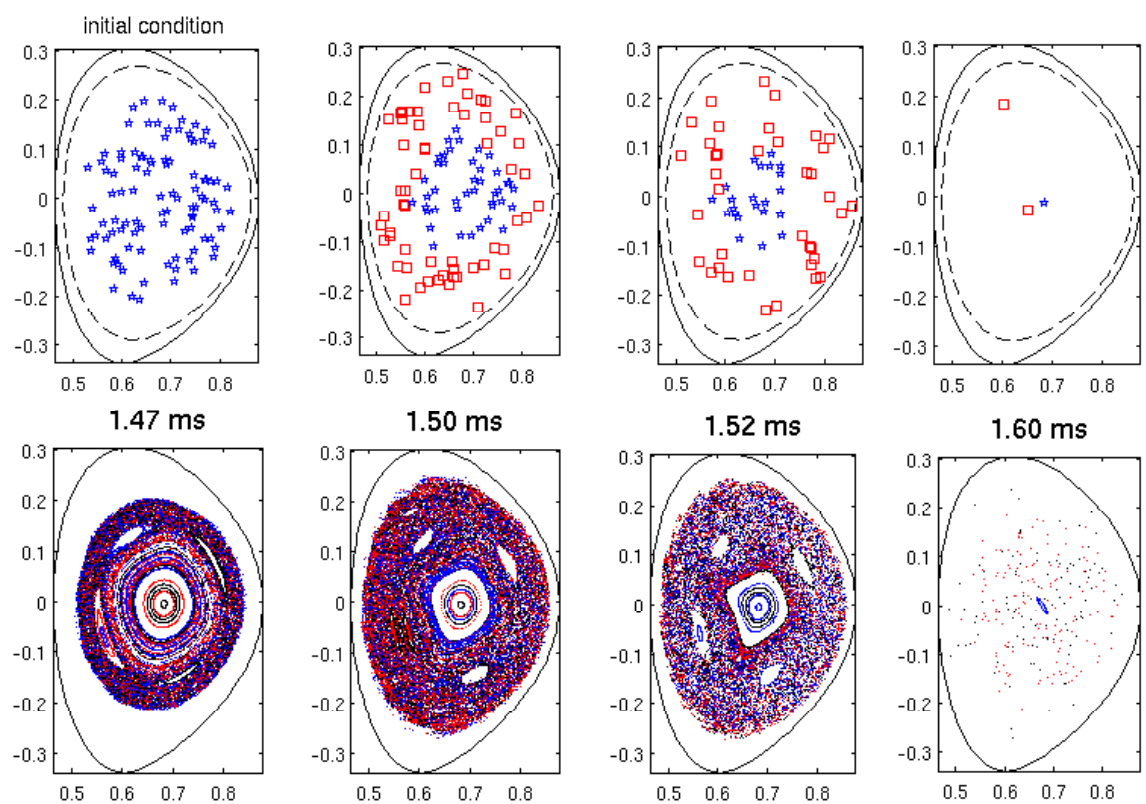
studying runaways is that no present device is as large as ITER (C-Mod is $1 / 9^{\text {th }}$ the size) and so Figure 12. Cross-sections of C-Mod from NIMROD MHD modeling in gas jet termination. Bottom row: magnetic flux surfaces become increasingly disordered as the impurity radiation forces cooling. Top row shows 100 trace suprathermal (blue) electrons accelerating to become runaways, (red) and then being lost by exiting through the last closed flux surface (dashed lines).

the runaway amplification factors are exponentially smaller. This shortcoming has been overcome in C-Mod by using the Lower Hybrid ( $\mathrm{LH}$ ) heating system to produce a target plasma where there is already a large population of hot electrons (not runaways), and then intentionally terminate the plasma with massive gas injection to examine the runaway formation as it would happen in ITER. Calculations show that if these electrons were confined, approximately half of the $1 \mathrm{MA}$ plasma current will reside in a runaway electron "tail" during the thermal quench. Indeed CMod diagnostics (figure 11) show the electrons do run away, however the fast electrons are lost by transport in $<1 \mathrm{~ms}$, and are absent by the time of the current decay. The 3-D

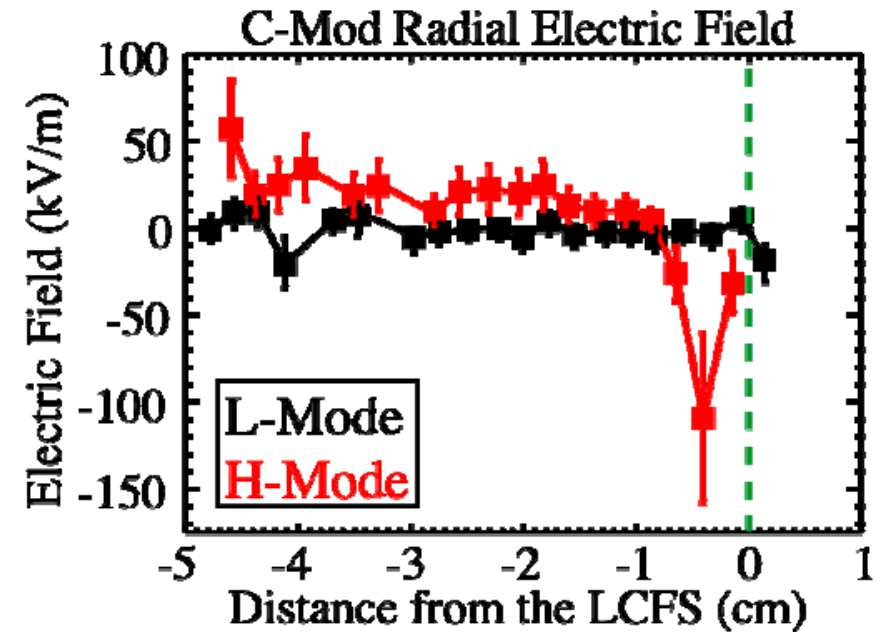

Figure 13: Comparison of radial electric field profiles in $L$ and $H$-mode. The width of the H-mode well is about 3 $\mathrm{mm}$.
NIMROD code, upgraded to include impurity radiation physics $\left[{ }^{16}\right]$ has been used to model fast electron confinement. Trace electrons were followed through the same 3-D code solution. Figure 12 shows that the original fast electrons from $\mathrm{LH}$ are indeed accelerated to multi $\mathrm{MeV}$ energy by the inductive increase in electric field during the thermal quench. Rapid growth of low-m coherent MHD causes B to become stochastic, and runaways are rapidly lost, in good agreement with experiment. These results strongly suggest that the same mechanism responsible for mitigation of radiated 
power concentration and halo currents also suppresses runaways, a very encouraging result for ITER.

Another issue for ITER is the toroidal distribution of the radiation emitted from a mitigated disruption. Even modest peaking factors (peak/average $\sim 2$ ) could cause localized melting of beryllium surfaces $\left[{ }^{17}\right]$ in ITER. Alcator C-Mod has used fast, $(100 \mathrm{kHz})$, AXUV diode arrays at multiple toroidal and poloidal locations to analyze the time and space evolution of radiation from a disruption induced by a mixed $\mathrm{He} / \mathrm{Ar}$ gas jet $\left[{ }^{18}\right]^{2}$. Prior to the thermal quench, a thin radiating layer localized to the gas-jet/plasma interface is shown to grow in time and expand around the plasma surface at approximately the neutral sound speed. At the same time, a toroidally symmetric feature on the emissivity profile is shown to propagate inward from the edge at $\sim 1 / 5$ the neutral sound speed. Both loss mechanisms are important in removing edge thermal energy. The radiation is highly asymmetric during the thermal quench, with brightness ratios of chords viewing towards and away from the gas jet varying from 0.3 to 3.0; however, integrated over the entire disruption event, the energy flux is shown to be uniform to within $\sim 25 \%$.

\section{Pedestal $E_{R}$ and $H$-Mode Dynamics}

The installation of new edge diagnostics has enabled detailed calculations of radial electric field $\left(E_{r}\right)$ in the C-Mod edge pedestal region. These measurements have shed new light into the connections between ExB shear suppression and H-mode confinement. Experiments were conducted to gain better control over H-mode densities, to explore new operating regimes, and to understand better the role that the edge pedestals play in these new operational spaces.

The radial electric field measurements made on C-Mod are qualitatively consistent with the idea that ExB shear is an essential piece of the H-mode puzzle. L-mode $\mathrm{E}_{\mathrm{r}}$ profiles are small in magnitude and relatively flat. In $\mathrm{H}$-mode, however, the electric field at the edge becomes

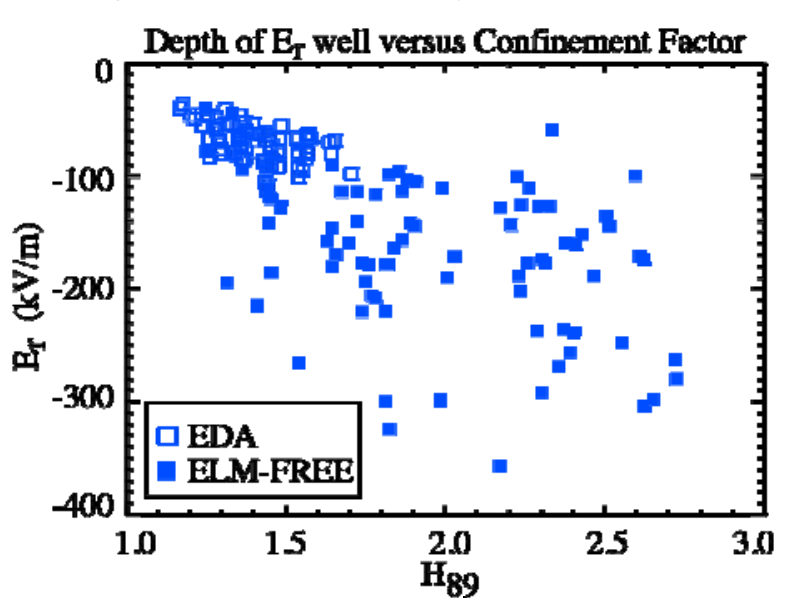

Figure 14. E $E_{r}$ well depth increases with improved confinement. sharply negative, creating a well-like structure and very strong $\mathbf{E x B}$ shear in the edge pedestal region (figure 13).

We track $\mathrm{E}_{\mathrm{r}}$ evolution through $\mathrm{H}$-modes in which there are significant changes to the quality of the plasma confinement, showing clear correlations. The depth of the $\mathrm{E}_{\mathrm{r}}$ well decays if $\mathrm{H}$-mode confinement degrades due to increases in radiated power (figure 14). Recent experiments have focused on understanding the effect of magnetic topology on edge pedestal structure and, in turn, its effect on plasma confinement. Pedestal characteristics are found to be sensitive to magnetic topology; improved

$\mathrm{H}$-mode confinement is obtained by operating near double-null. In these configurations plasmas with improved temperature but moderate density pedestals were obtained and maintained for many confinement times.

\section{Improved L-Mode}


It has long been observed that the threshold power for triggering the transition to H-mode is strongly affected by divertor topology; for the single-null configuration, changing the direction of the ion $\mathrm{BX} \nabla \mathrm{B}$ drift can lower the threshold by as much as a factor of two (for ion drift toward the X-point compared to away from the X-point). $\left[{ }^{19}\right]$ Previous experiments

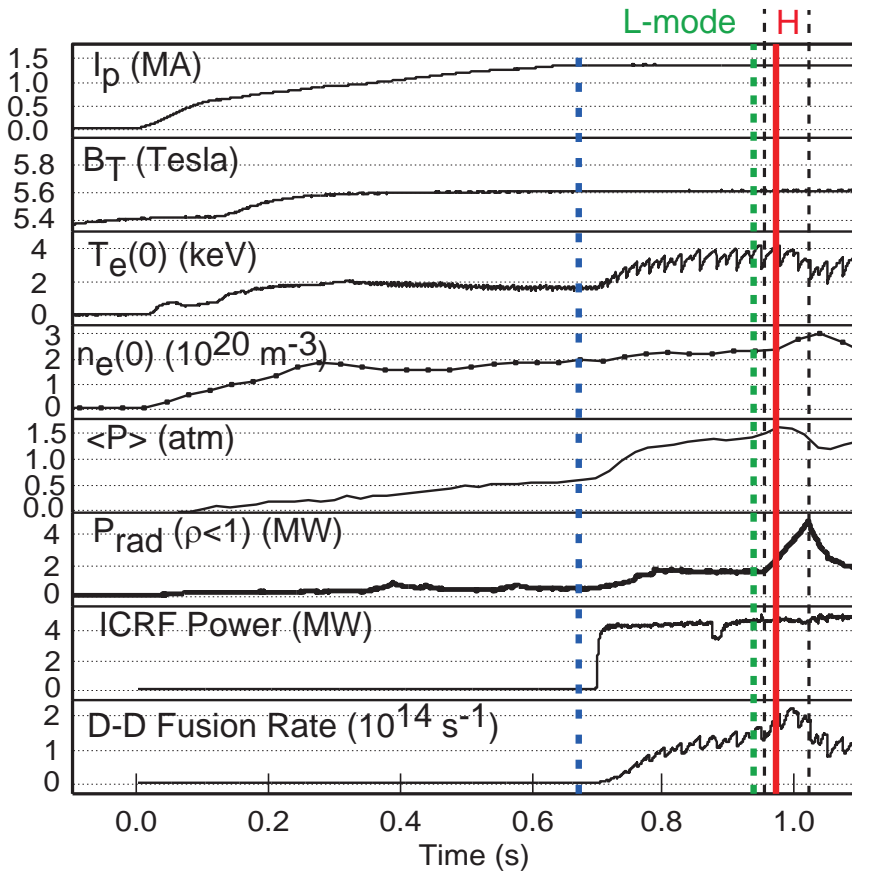

Figure 15. Time histories of plasma parameters for an improved L-mode discharge. For this upper single null divertor case, the ion drift direction is down, away from the active X-point. The H-mode threshold from the ITER scaling is $1.8 \mathrm{MW}$. Other parameters for this discharge: $q_{95}=2.9$, triangularity $=0.6$.

plasma current (low safety factor), with strong shaping and high auxiliary heating power (ICRF). Time histories of plasma parameters from a typical discharge are shown in figure 15. The total input power during the ICRF heating is more than three times the $\mathrm{H}$-mode threshold as predicted by the ITER scaling. $\left[{ }^{23}\right]$ In spite of this, the complete transition to $\mathrm{H}$-mode, with the development of a strong density pedestal, is delayed by about $4 \tau_{\mathrm{E}}$. During this time, a strong temperature pedestal forms, and global confinement is $1 \mathrm{x} \tau_{\text {ITER-98-Y2 }}$; following ASDEX-U $\left[{ }^{24}\right]$ we refer to this phase of the discharge as an improved L-mode. During this phase, there is no edge particle barrier, eliminating any issues with impurity build-up and radiated power. Eventually the plasma enters an ELM-free H-mode phase, with the formation of a strong density pedestal, and rapid increase in core impurities. Edge temperature and density profiles are shown in figure 16 for three phases of the discharge (times of the profiles are shown as on C-Mod have investigated the relationships among spontaneous rotation $\left[{ }^{20}\right]$, strong flows in the scrape-off-layer on open field lines toward the divertor[ $\left.{ }^{21}\right]$, magnetic topology and the threshold. The SOL flows provide a boundary condition for the flows seen on closed field lines, and at least partially explain the zeroth order effect on the H-mode power and edge temperature threshold $\left[{ }^{22}\right]$. More recent C-Mod experiments have sought to exploit the increased threshold for transition to the traditional H-mode, with its associated fast formation of both temperature and density barriers at the plasma edge, by running in the unfavorable drift direction (away from the active X-point), at high

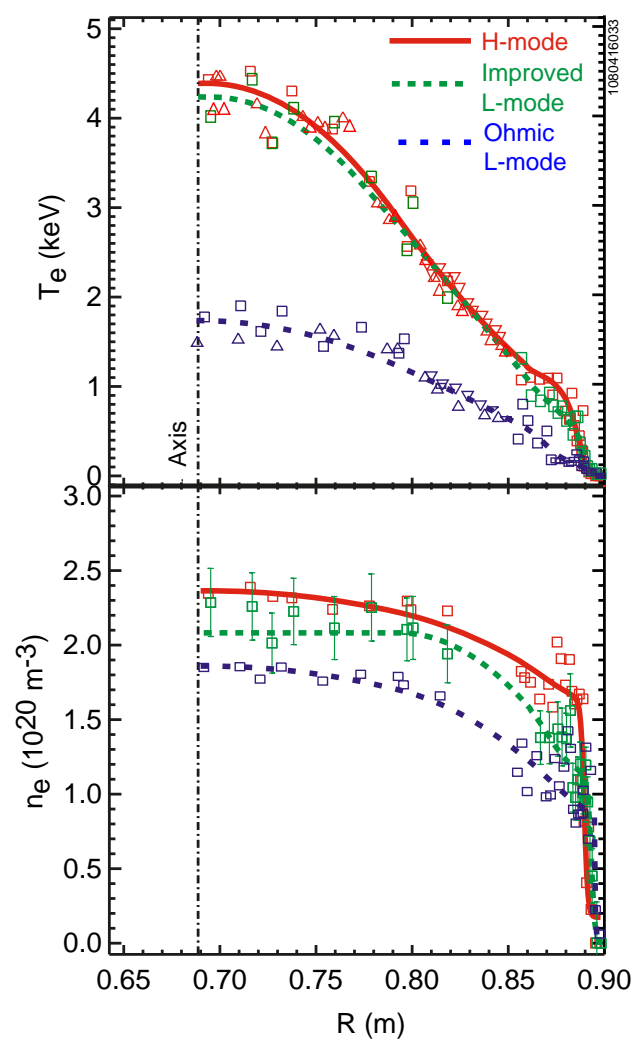

Figure 16. Radial profiles of temperature and density, comparing ohmic L-mode, improved L-mode and $H$-mode. 
colored vertical lines in figure 15): ohmic L-mode, improved L-mode, and shortly after the transition to H-mode. The formation of the strong temperature barrier is evident during the improved L-mode phase, while the edge density gradient remains close to that of the ohmic L-mode. The combination of good global energy confinement, no ELMs, and no impurity accumulation, could be a very favorable scenario for ITER operation, especially if the transition to full $\mathrm{H}$-mode can be avoided. ITER plans to operate in a regime with relatively small margin of input power above the expected H-mode threshold (for the favorable ion drift direction), which makes the improved L-mode even more interesting for further research.

\section{References}

${ }^{1}$ Y. Lin, et al., submitted to Phys. Rev. Lett. (2008).

${ }^{2}$ E. Nelson-Melby, et al., Phys. Rev. Lett. 90, 155004 (2003).

${ }^{3}$ E.F. Jaeger, et al., Phys. Rev. Lett. 90, 195001 (2003).

${ }^{4}$ J.R. Myra and D.A. D'Ippolito, Phys. Plasmas 9, 3867 (2002).

${ }^{5}$ E.F. Jaeger et al., Phys. Plasmas 15, 072513 (2008).

${ }^{6}$ J. Myra et al., Nuclear Fusion 46, S455 (2006).

${ }^{7}$ B. Lipschultz, et al., Journal of Nuclear Materials, 363-365, 1110 (2007).

${ }^{8}$ W. Eckstein, J. Bohdansky, and J. Roth, Atomic and Plasma-Material Interaction Data for Fusion, suppl. Nuclear Fusion 1, 51 (1991).

${ }^{9}$ E. Y. Wang et al., J. Applied Physics 61, 4786 (1987).

${ }^{10}$ R. W. Harvey and M. McCoy, in Proceedings of the IAEA Technical Committee Meeting on Simulation and Modeling of Thermonuclear Plasmas, Montreal, Canada, (1992), USDOC NTIS Document No. DE93002962.

${ }^{11}$ A. Ince-Cushman, et al., Phys. Rev. Lett. (2008) in press.

${ }^{12}$ V. Pericoli Ridolfini, et al., AIP Conference Proceedings, n 595, 245 (2001).

${ }^{13}$ B. Lipschultz, et al., Physics of Plasmas, 13, 56117 (2006).

${ }^{14}$ R.S. Granetz, D.G. Whyte, V.A. Izzo, et al., Nucl. Fusion 46, 1001 (2006).

${ }^{15}$ R.S. Granetz, E.M Hollmann, D.G. Whyte, V.A. Izzo, et al., Nucl. Fusion 47, 1086 (2007).

${ }^{16}$ V. Izzo, et al., Physics of Plasmas, 15, 056109 (2008).

${ }^{17}$ D. Morozov, et al., Plasma Phys. Control. Fusion 49, 929 (2007).

${ }^{18}$ M.L. Reinke, et al., Toroidally Resolved Radiation Dynamics During a Gas Jet Mitigated Disruption on Alcator C-Mod, submitted to Nucl. Fusion (2008).

${ }^{19}$ A.E. Hubbard, J.W. Hughes et al., Phys. Plasmas 14, 056109 (2007).

${ }^{20}$ J.E. Rice, et al., Physics of Plasmas, 11, 2427 (2004)

${ }^{21}$ B. LaBombard, et al., Nuclear Fusion, 44, 1047 (2004)

${ }^{22}$ B. LaBombard, et al., Physics of Plasmas, 12, 56111-1 (2005)

${ }^{23}$ J.A. Snipes, et al., Nuclear Fusion, 36, 1217 (1996)

${ }^{24}$ F. Ryter, W. Suttrop, B. Brusehaber, et al., Plasma Phys. Control. Fusion 40, 725 (1998). 